\title{
Deux lettres inédites de Jean Jaurès à Eduard
} Bernstein

\section{Jean-Numa Ducange}

\section{(2) OpenEdition \\ 1 Journals}

\section{Édition électronique}

URL : https://journals.openedition.org/ahrf/11770

DOI : 10.4000/ahrf. 11770

ISSN : 1952-403X

Éditeur :

Armand Colin, Société des études robespierristes

\section{Édition imprimée}

Date de publication : 1 juin 2010

Pagination : 219-225

ISBN : 978-2-200-92632-8

ISSN : 0003-4436

\section{Référence électronique}

Jean-Numa Ducange, "Deux lettres inédites de Jean Jaurès à Eduard Bernstein », Annales historiques de la Révolution française [En ligne], 360 | avril-juin 2010, mis en ligne le 01 juin 2013, consulté le 24 avril 2022. URL : http://journals.openedition.org/ahrf/11770 ; DOI : https://doi.org/10.4000/ahrf. 11770 


\section{DEUX LETTRES INÉDITES DE JEAN JAURÈS À EDUARD BERNSTEIN ${ }^{1}$}

Jean-Numa DUCANGE

Les lecteurs du quatrième tome de l'Histoire socialiste de la Révolution française de Jean Jaurès «La Révolution française et l'Europe » savent que le social-démocrate allemand Eduard Bernstein, connu pour avoir révisé l'orthodoxie marxiste de la social-démocratie allemande à la fin du dix-neuvième siècle, a fourni une aide documentaire au socialiste français pour la partie de son œuvre consacrée à l'Allemagne :

" Je signalai le passage à Edouard Bernstein, qui a cherché et trouvé le livre à la Bibliothèque royale de Berlin. Il en a publié dans le $3^{\text {ème }}$ cahier de ses Documents du Socialisme la partie communiste $»^{2}$.

Bernstein a en effet publié, suite à la demande de Jean Jaurès, un texte sous le titre « Aus einer deutschen Kommunistischen Schrift von 1792. Der Mensch und seineVerhältnisse »dans sa revue Dokumente des Sozialismus ${ }^{3}$ qui fut traduit et repris dans I'Histoire socialiste. On sait depuis que ce « communiste allemand » est Carl Wilhelm Fröhlich ${ }^{4}$.

En dehors de cet échange qui, dans l'édition d'Albert Soboul, n'était pas plus détaillé faute d'accès aux documents, la réception de

(1) Merci à Gilles Candar de m'avoir incité à rechercher ces documents.

(2) Jean JAURĖs, Histoire socialiste de la Révolution française, Paris, Éditions Sociales, 1969, tome IV, p. 274.

(3) Dokumente des Sozialismus, Hefte für Geschichte, Urkunden und Bibliographie des Sozialismus, 1902, p. 114-131. Éditée à Berlin de 1901 à 1905, la revue avait notamment pour objectifs de publier des manuscrits rares ou inédits de l'histoire du socialisme. C'est dans cette revue que furent publiés les premiers fragments de L'Idéologie allemande de Karl Marxet Friedrich Engels dont l'intégralité ne sera connue qu'en 1932.

(4) Gerhard Steiner, "Un utopiste allemand du $18^{\text {ème }}$ siècle : Carl Wilhelm Fröhlich », Annales historiques de la Révolution française, $n^{\circ} 166,1961$, p. 449-460. 
I'Histoire socialiste en Allemagne se résume souvent à la critique acerbe deFranz Mehring publiée dans la NeueZeiten 1903 et traduiteà l'époque danslarevueLemouvementsocialiste ${ }^{5}$.PourtantBernstein, au-delàd'une aidedocumentaire ponctuelle, a cherchéà promouvoirla grande fresque jaurésienneen Allemagneen rendant comptedans lequotidien berlinois du parti social-démocrate Vorwärts et dans sa revue Dokumente des Sozialimus ${ }^{6}$. Dans cette dernière sont aussi parus de courts extraits de I'Histoire socialiste de Jaurès. Les débats suscités par la publication de l'Histoire socialiste en Allemagne ne sauraient donc se résumer à la seule approche de Mehring.

Nous souhaiterions présenter ici deux lettres, inédites à notre connaissance, de Jaurès à Bernstein. Elles éclairent certains aspects méconnus de la réception de l'ouvrage de Jaurès qui, il faut le rappeler, n'a jamais été traduit en allemand à l'exception de courts extraits.

La première est issue des archives privées d'Eduard Bernstein conservées à I'Institut international d'histoire sociale d'Amsterdam (IISG). D'un intérêt essentiellement documentaire, elle date d'octobre 1901 et constitue la demande de Jaurès pour le document recherché pour I'Histoire socialiste. Elle est classée sous la référence D 307.

La seconde, plus développée et originale, serait inédite jusqu'à une date récente. Nous l'avons consultée dans le fonds « France, various manuscripts Collection » à l'IISG récemment classé en 2008. II regroupe unensemble de documentsvariés demanuscritsfrançais, notamment de Jean Jaurès, sans que la provenance soit connue. Cette lettre, qui porte la référence « Jaurès 12 » devait probablement faire partie initialement du fonds Bernstein 7 . L'année n'est pas indiquée, mais il s'agit du 19 janvier 1902 puisqu'elle fait suite à l'envoi du premier volume. Karl Kautsky qui a aussi reçu le volume date en effet sa lettre de remerciements de décembre $1901^{8}$. Aucune lettre de Jaurès à Kautsky n'a suivi cet envoi ce qui rend cet échange avec Bernstein d'autant plus singulier.

(5) Sur cette question voir Irmgard HARTIG, «Observations sur la querelle entre Jaurès et Mehring, » Annales historiques de la Révolution française, n²11, 1973, p. 112-127.

(6) Ces comptes rendus seront prochainement mis en ligne sur le site de l'Institut d'histoire de la Révolution française (http://ihrf.univ-paris1.fr/)

(7) Madeleine Rebérioux cite une phrase de cette lettre sans indiquer la référence précise qui lui correspond (Madeleine RebéRIoux, "Jaurès, historien de la Révolution française » dans Jaurès, historien de la Révolution française, Castres, Centre national et Musée Jean Jaurès, 1989, p. 40).

(8) Lettre de Karl Kautsky à Jean Jaurès (en français), IISG, Archives Karl Kautsky, C 455, 19 décembre 1901. 
Sisa rédaction a étécertainement motivée par le long compterendu de Bernstein dans le Vorwärts le 12 janvier 1902, on peut se demander pourquoi Jaurès s'adresse à Bernstein pour introduire son Histoire socialiste en Allemagne. Le contexte politique n'était pas des plus favorables. Bernstein avait publié des articles, regroupés ensuite dans un ouvrage paru en 1899, qui révisaient les fondements de la doctrine du parti social-démocrate allemand ${ }^{9}$ - d'où l'étiquette de « révisionnisme » - en critiquantnotammentledécalageentrelesprincipesrévolutionnairesproclamés et la réalité politique d'une social-démocratie devenue pragmatique ("Qu'elle ose paraître ce qu'elle est! »). Le débat est virulent et a des échos jusqu'en France : on sait que Jaurès a critiqué la révision de Bernstein dans Bernstein et l'évolution de la méthode socialiste ${ }^{10}$ : cette conférence montre qu'il a néanmoins un point de vue équilibré sur cette question. « Ni orthodoxe, ni révisionniste, ni radical. Inclassable »"11 selon Madeleine Rebérioux. Le dialogue avec les « révisionnistes » dont Bernstein est le représentant le plus éminent n'a en effet jamais été rompu. Jaurès cherchedesappuis danslepartiallemand dontle point devueétait essentiel pour les socialistes français sur la question « ministérielle $»^{12}$.

Ces lettresà Bernstein trouvent un complément utile dans d'autres extraits de correspondances avec divers sociaux-démocrates allemands avec qui Jaurès correspond. Citons une longue lettre adressée à Vollmar, partisan des thèses de Bernstein, dans laquelle Jaurès expose sa conception de la participation gouvernementale :

" J'aurais été bien heureux de pouvoir assister à Munich à l'important Congrès de la démocratie socialiste allemande et de répondre à la si aimable invitation que Madame Vollmar et vous m'adressez. Mais cela m'est matériellement impossible. Mes « vacances » sont et doivent être presque entièrement consacrées à liquider un énorme arriéré de travail.II

(9) Eduard Bernstein, Die Voraussetzungen des Sozialismus und die Aufgaben der Sozialdemokratie, Stuttgart, Dietz, 1899, 188 p. (en français, Edouard BERNSTEIN, Les présupposés du socialisme, Paris, Seuil, 1974, 300 p.)

(10) Jean JAURĖs, « Bernstein et l'évolution de la méthode socialiste » (1900), Études socialistes 1897-1901, t. II, 1931 (réimpression 1995), p. 117-139. Voir aussi les remarques d'Emmanuel Jousse, Réviser le marxisme? d'Édouard Bernstein à Albert Thomas, 1894-1914, Paris, L'Harmattan, 2007. II ne traite pas cependant du lien avec l'écriture de l'Histoire socialiste.

(11) Madeleine RebÉRIOux, "Jaurès le marxisme », Histoire du marxisme contemporain, t. 3, 1977, p. 237.

(12) Le 22 juin 1899, Alexandre Millerand devient ministre du Commerce dans le gouvernement Waldeck-Rousseau; premier socialiste participant à un gouvernement « bourgeois » il est soutenu par Jaurès. 
faut que je mène jusqu'au bout l'histoire de la Convention - et pour deux ou trois mois encore cela m'accable. Je me hâte d'arriver au terme afin de retrouver ensuite toute ma liberté d'action pour les luttes immédiates, mais je ne veux pas lâcher un travail qui m'a coûté dix ans de recherche $[\ldots] \gg^{13}$.

Au-delà des enjeux politiques, des motivations plus proprement historiennes rapprochent Jaurès et Bernstein : celui-ci a écrit une histoire de la Révolution anglaise et des niveleurs que Jaurès connaissait ${ }^{14}$. Bernstein avait aussi traduit en allemand et préfacé en 1887 des articles de Gabriel Deville sur Babeuf et la conjuration des égaux regroupés pour l'occasion sous forme d'ouvrage ${ }^{15}$. Jaurès s'adressait ainsi au socialdémocrate le plus familier des travaux historiques de ce type; Karl Kautsky n'avait par exemple jamais effectué un travail aussi approfondi et en était resté à des généralisations théoriques.

Nous remercions I'IISG d'Amsterdam de nous autoriser à publier ici les deux lettres ci-dessous. Ces correspondances paraîtront dans le tome VIII des CFuvres de Jean Jaurès: Défense républicaine et participation ministérielle 1899-1902 édité par Maurice Agulhon et JeanFrançois Chanet chez Fayard. Toutes les notes sont de nous. Les passages soulignés ici le sont par Jaurès.

Jean-Numa DuCANGE

Docteur en histoire Université de Rouen - Département d'histoire Rue Thomas-Becket 76130 Mont-Saint-Aignan

jean-numa.ducange@univ-rouen.fr 1902.

(13) Lettre de Jean Jaurès à Georg von Vollmar, IISG, archives Vollmar, 1027, 3 septembre

(14) Eduard BERNSTEIN, Kommunistische und demokratisch-sozialistische Strömungen während der englischen Revolution, J.H.W. Dietz, Stuttgart, 1895, 367 p.

(15) Gabriel DevilLE, Gracchus Babeuf und die Verschwörung der Gleichen, GöttingenZurich, Sozialdemokratische Bibliothek, 1887, 72 p. 
7 avenue des Chalets

Passy - Paris

le samedi 19 octobre

\section{Cher citoyen}

Excusez, je vous prie, mon indiscrétion. Mais vous pouvez me rendre un très grand service. J'étudie en ce moment l'action immédiate de la Révolution française sur les idées politiques et sociales de l'Allemagne. Or, je trouve dans la correspondance de Goerg Forster (Neunter Band, Briefwechsel, Seite 77) l'indication suivante qui me paraît très intéressante :

"EineanderegrosseFreudegewährtemirgesterneingutesdeutsches Buch: Über den Menschen und seine Verhältnisse, 1792, Kl. Octav. Berlin, in der Frankeschen Buchhandlung. Es ist eins der seltenen Produkte unserer Zeit, das Werk eines jungen aber sehr richtig denkenden und empfindenden Menschen. Ich müsse wissen, wer er ist und wie er heisst. Einiges, wie wir denn unmöglich immer zusammentreffen können, ist meiner Ansicht fremd, besonders seine politischen Ideen von Gemeinschaft des Eigenthums. ${ }^{16}{ }^{~}$ Cet écrivain parle aussi dans son livre de Mayence (Mainz). J'aimerais bien, moi aussi, savoir ce qu'est ce livre. Je vous serais bien reconnaissant de le faire rechercher pour moi à Berlin ou de m'en procurer les extraits les plus importants. Je voudrais savoir surtout, avec ses idées sur le communisme, comment iljuge la Révolution française et quelle influence elle a eue sur lui.

Excusez-moi, je vous prie, mais vous comprenez sans peine l'intérêt que j'attache à cette recherche.

En retour, si je puis faire pour vous à Paris, à la Bibliothèque nationale ou ailleurs, une recherche utile, je suis tout à votre disposition.

Croyez, je vous prie, à ma bien vive sympathie.

Jean Jaurès.

(16) Le passage est traduit par Jaurès dans I'Histoire socialiste (t. IV, p. 274) : « Un bon livre allemand me réservait hier une autre joie : Sur I'homme et sa condition [...] à la librairie de Franke. C'est une des plus rares productions de notre temps, l'œuvre d'un homme jeune, qui pense et sent avec justesse. Je voudrais savoir qui il est et comment il se nomme. Comme il est impossible qu'il y ait accord complet des esprits, il y a un point sur lequel ses vues s'éloignent des miennes : ce sont ses idées politiques sur la communauté de la propriété ». 
7 avenue des Chalets,

Passy - Paris

Le 25 janvier

\section{Au citoyen Bernstein}

Je serais très heureux que vous publiiez dans les Dokumente ${ }^{17}$ ou les Monatshefte ${ }^{18}$ tout ce qui de mon livre pourra intéresser vos lecteurs. Je vous suis très reconnaissant aussi de vouloir bien le faire traduire en allemand - et je m'en remets tout à vous du suivi de trouver un traducteur compétent et consciencieux.

J'ai été bien content de votre lettre et de l'article du Vorwärts ${ }^{19}$. J'ai fait mon travail en remontant toujours aux documents originaux et aux sources-etlaméthodedumatérialismehistoriquequandonnelecompromet point par de véritables enfantillages comme l'ont fait chez nous Lafargue ${ }^{20}$ et chez vous Mehring ${ }^{21}$, est un guide excellent.

Le second volume, qui est aussi de moi, La Législative va paraître et je vous l'enverrai dans quelques jours. Le troisième volume, La Convention, paraît en ce moment en livraisons. Elle paraîtra en volume dans huit ou dixmois. C'est Guesdequi devaitécrire la Convention mais en a étéempêché par son état de santé, du moins à ce qu'il m'a dit. C'est moi encore qui la rédige : et j'aurai aussi conduit la Révolution française jusqu'au 9 thermidor. Si je n'avais pas été soutenu par une longue préparation et continué depuis dix ans, j'aurais succombé sous le fardeau. J'espère que l'ensemble sera substantiel etlié. Àmesurequej'aiapprofondietà mesure aussiquese développentlesévénements dela révolution,j'aiconstatéun éveil plus vif et une formation plus précise de la conscience prolétarienne sous l'action de la démocratie. Et j'essaie de saisir les premiers points d'attache de l'extrême démocratie révolutionnaire et du socialisme. Je vous enverrai dans un mois les livraisons de la Convention où je cite les opuscules de l'Ange qui à Lyon en 1790, 1792 et 1795, a formulé toute la

(17) II s'agit des Dokumente des Sozialismus. Voir note 2.

(18) Il s'agit de la revue Sozialitische Monatshefte créée en 1897 où s'exprime le point de vue « révisionniste ». Dirigée par Joseph Bloch, elle a traduit plusieurs articles politiques de Jaurès.

(19) Un long compte rendu de Bernstein a paru dans le Vorwärts le 12 janvier 1902.

(20) Paul Lafargue (1842 - 1911), guesdiste, est un des principaux adversaires de Jaurès dans la mouvance socialiste.

(21) Jaurès fait allusion à ici à l'ouvrage de Franz Mehring (1846 - 1919) sur « La légende de Lessing » (Franz Merring, Die Lessing Legende, Stuttgart, Dietz, 1893, 426 p.) qu'il critiquera dans son tome sur la Convention pour sa méthode qu'il juge inspirée d'un matérialisme historique étroit. Voir note 4. 
doctrine de Fourier avec une précision extraordinaire. J'ai donné aussi les moyenstermesentreladémocratierobespierristeetlebabouvisme.Jeme permets devous dire toutcela non pas pourvous surcharger de ma propre besogne (vous avez bien assez de la vôtre) mais pour bien vous direà quel point je suis, avec vous un socialiste démocrate.

Je suis bien heureux de l'action que vous exercez en Allemagne et j'ai hâte de vous voir au Reichstag 22 .

Merci encore et à bien à vous

Jaurès

(22) Bernstein est élu en 1902 député au Reichstag et le reste jusqu'en 1907, puis de nouveau de 1912 à 1918 et enfin de 1920 à 1928. 